\title{
Site-specific integration of transgene targeting an endogenous lox-like site in early mouse embryos
}

\author{
Masanori Ito • Keitaro Yamanouchi • Kunihiko Naito • \\ Michele P. Calos • Hideaki Tojo
}

Received: 28 May 2010 /Revised: 25 September 2010 / Accepted: 27 September 2010 / Published online: 26 November 2010

(C) Institute of Plant Genetics, Polish Academy of Sciences, Poznan 2010

\begin{abstract}
Functional lox-like sequences have been identified within the yeast and mammalian genome. These hetero-specific lox sites also allow Cre recombinase to specifically target efficient integration of exogenous DNA into the endogenous pseudo-lox ( $\psi l o x$ ) sequences that occur naturally in the host genome using a Cre/loxP integrative recombination system. We investigated whether the Cre/ $\psi$ lox system is useful for site-specific integration of transgenes and for improving the production efficiency of transgenic animals. This is the first report on Cre-mediated integrative recombination targeting an endogenous lox-like sequence termed pseudo-loxm5 ( $\psi$ loxm5) in early mouse embryos. We characterized the Cre/ $\psi$ loxm 5 system in embryonic environment. Cre-expressing plasmid and a transgene (CMV/LacZ gene) flanked by $\psi$ loxm 5 and uloxcorem 5 sites were co-microinjected into the pronucleus
\end{abstract}

M. Ito $(\bowtie) \cdot \mathrm{K}$. Yamanouchi $\cdot$ K. Naito $\cdot$ H. Tojo

Laboratory of Applied Genetics, Graduate School of Agricultural and Life Sciences, The University of Tokyo,

1-1-1 Yayoi, Bunkyo-ku,

Tokyo 113-8657, Japan

e-mail: itomasanori@med.toho-u.ac.jp

\section{P. Calos}

Department of Genetics, School of Medicine, Stanford University, Stanford, CA 9405-5120, USA

H. Tojo

Yamazaki College of Animal Health Technology,

4-7-2 Minamioosawa, Hachioji,

Tokyo 192-0364, Japan

Present Address:

M. Ito

Department of Pharmacology, School of Medicine,

Faculty of Medicine, Toho University,

5-21-16 Omori-Nishi, Ota-ku,

Tokyo 143-8540, Japan of fertilized mouse oocytes. The injected eggs were transferred into foster mothers, and the recombination products were investigated. The results show that the $\psi$ loxm 5 site is an active substrate for Cre-mediated recombination in the mouse embryonic environment. The transgenesis efficiency was up to $27 \%(6 / 22)$. The site-specific integration of the transgene into the endogenous $\psi$ loxm 5 site was found in $50 \%$ of the transgenic pups. Our findings demonstrated that the Cre/ $\psi$ loxm 5 integrative recombination system is an efficient and simple strategy for targeting an endogenous lox-like site in mammalian embryos.

Keywords Cre $\cdot$ Microinjection $\cdot$ Pseudo-lox $\cdot$ Site-specific integration - Transgenic animal

\section{Introduction}

Transgenic animals generated by standard DNA microinjection methods show an extreme variability in expression of the introduced gene. This variability is a result of the position effects on gene expression caused by random integration of the transgene into the host genome, and from the highly variable copy numbers of the integrated foreign gene. Further, the efficiency of the transgenesis process has been intolerably low, particularly in farm animals. An appealing strategy to circumvent such constraints would involve site-specific integration of the introduced sequences into an appropriate position in the genome. Homologous recombination can serve as an alternative for site-specific DNA recombination, but its efficiency is low (Vega 1991). The Cre/loxP recombination system is highly specific and efficient in eukaryotic cells. Cre has been shown to efficiently carry out intra-molecular and inter-molecular recombination in both prokaryotic and eukaryotic cells 
(Sauer 1987; Sauer and Henderson 1988; Sauer and Henderson 1990). Furthermore, Cre has been reported to catalyze recombination at $\operatorname{lox} P$ sites having a certain degree of homology with the wild-type loxP (Lee and Saito 1998; Sauer 1992; Sauer 1996; Thyagarajan et al. 2000). Mutant lox sites have been developed to increase the efficiency of Cre-mediated insertion to avoid re-excision (Albert et al. 1995; Araki et al. 1997; Liu et al. 2007; Chatterjee et al. 2010; Kameyama et al. 2010).

Recombinase-mediated integration would be more useful for genetic engineering of animals if the sites that could act as the targets for the Cre recombinase occurred endogenously at appropriate locations in the mammalian genome such as in regions outside of the functional endogenous genes. Such a strategy would likely result in iso transgenic lines having identical expression levels of the transgene. Functional cryptic lox sites have been shown to occur naturally in the genome of yeast (Sauer 1996), human, and mouse (Thyagarajan et al. 2000). An illegitimate Credependent chromosomal rearrangement has been reported in the genome of a Cre transgenic mouse in the absence of the loxP sequences (Schmidt et al. 2000). Furthermore, it has been reported that chromosome analysis after Cre expression in mammalian cells revealed numerous chromosomal aberrations and an increased number of sister chromatid exchanges (Loonstra et al. 2001). Such genomic rearrangement strongly suggests the presence of functional cryptic lox sites in the mouse genome.

We focused on the endogenous $\psi$ loxm 5 , which has been identified in the mouse genome and has been shown to function in Cre-mediated recombination assays performed in bacteria and cultured mammalian cells (Thyagarajan et al. 2000). In this study, we investigated whether the Cre/ $\psi$ loxm 5 system is useful for site-specific and stable integration of the trangene in mice. Here, we show an efficient and simple strategy for introducing a foreign gene into the mammalian genome, targeting an endogenous lox-like site in mouse embryos using the Cre/loxP integrative recombination system.

\section{Materials and methods}

Plasmid construction

Three kinds of plasmids were used in the present study. The Cre-expressing plasmid, pCAG/NCre (Sakai and Miyazaki 1997), containing the bacteriophage P1 Cre gene driven by the $\mathrm{CAG}$ (cytomegalovirus immediate-early enhancer, chicken $\beta$-actin promoter and fusion intron of chicken $\beta$-actin and rabbit $\beta$-globin) promoter (Niwa et al. 1991), and including the polyadenylation signal (Fig. 1b), was the kind gift of Dr. I. Saito (The University of Tokyo, Japan). The reporter plasmid (pCMV/loxP/LacZ) (Fig. 1a) was constructed by inserting an arbitrary $1.5-\mathrm{kb}$ bacterial sequence, as a stuffer, at the Swa I site between the two loxP sites of the UL WL plasmid (RGB DNA Bank, Japan). The resulting loxP flanked stuffer was excised and ligated at the EcoRI/SalI sites of the pCMV-SPORT- $\beta$ gal (Life Technologies, Tokyo, Japan). Pseudo-loxm5 plasmid (p $\psi$ loxm5), containing the ßgal-expressing gene ( $L a c Z$ ) driven by cytomegalovirus (CMV) promoter, was used. The CMV/LacZ construct was flanked by two $\psi$ lox sequences: $\psi$ loxm 5 and $\psi$ loxcorem 5
Fig. 1 Cre-transient expression, recombination, and $\mathrm{X}$-gal staining of early-stage embryos. The structure of the target plasmid (pCMV/loxP/LacZ) (a) and its recombination form (c) are shown. The lac $Z$ gene is silent before recombination. After expression of $\mathrm{pCAG} / \mathrm{NCre}$ (b) in fertilized eggs. Cremediated recombination induces deletion of a floxed stuffer fragment and results in the expression of lacZ gene. pCMV/ loxP/LacZ and pCAG/NCre were co-microinjected into the pronucleus of mouse fertilized eggs. The surviving eggs were stained with X-gal after $10 \mathrm{~h}$ of culturing (d) a) $\mathrm{pCMV/loxP/LacZ}$

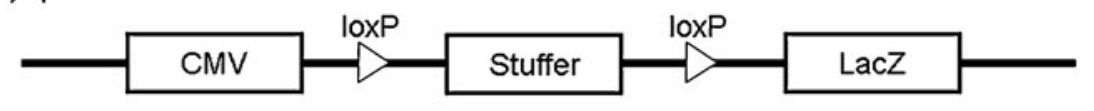

b)

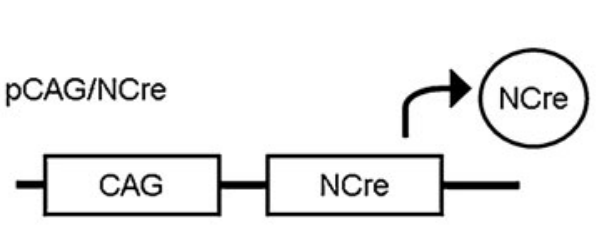

c) After recombination

d)

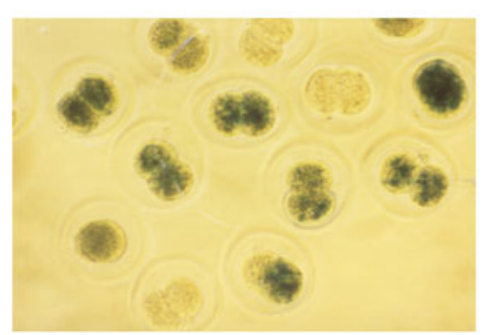


a) Endogenous $\Psi$ loxm5 in mouse genome

b) $\mathrm{p} \Psi \operatorname{loxm} 5$

c) PCAGNCre

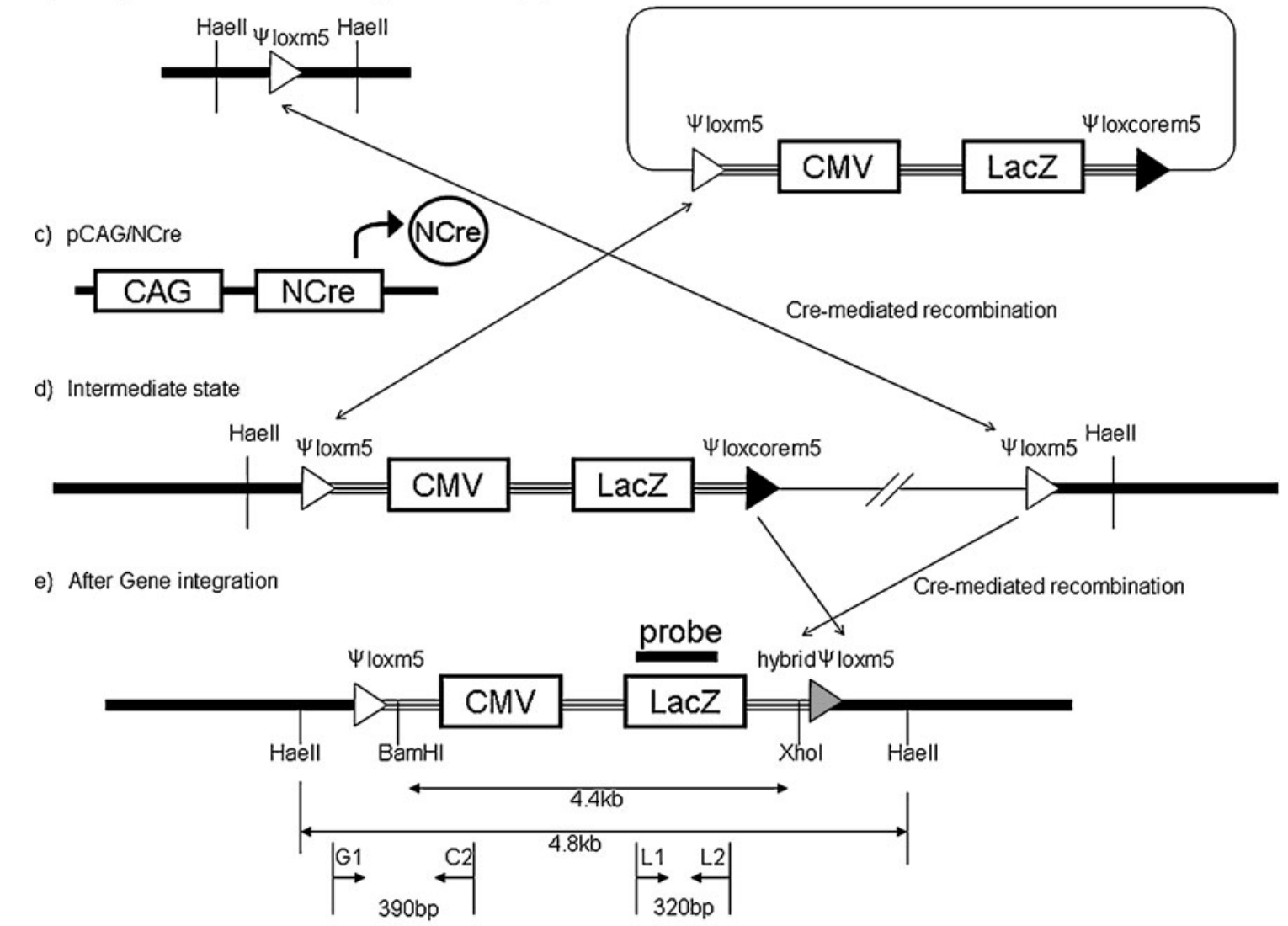

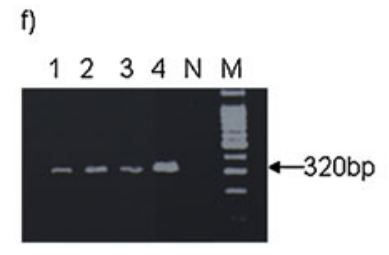

j)

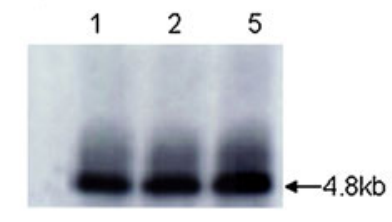

g)

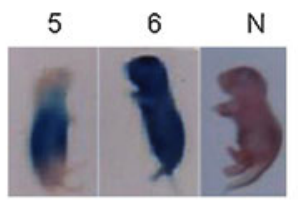

h)

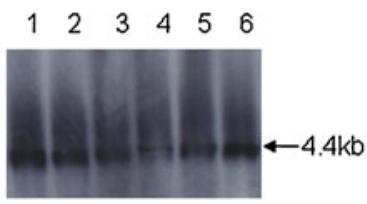

i)

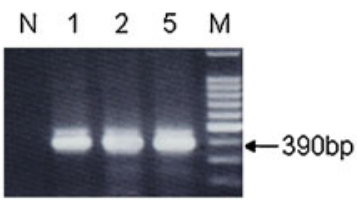

k)

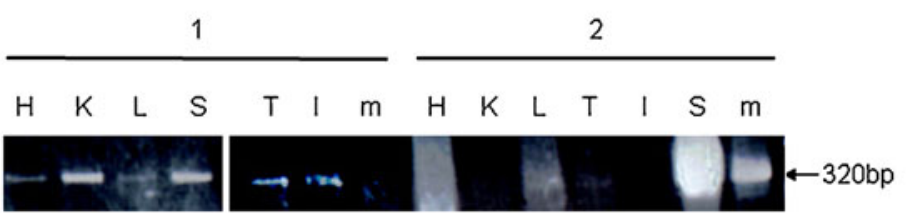

Fig. 2 Strategy and analysis for targeting the endogenous $\psi$ loxm5 site. The structure of the targeted $\psi$ loxm 5 locus (a) and the plasmid pwloxm5 (b), and their recombination forms (d, e) are shown. pyloxm5 and Cre-expressing plasmid (pCAG/NCre) (c) were comicroinjected into the pronucleus of mouse fertilized eggs. In the first step, Cre-mediated recombination results in insertion of the transgene, containing its plasmid backbone (d). In the second recombination, Cre mediates single-copy integration of the transgene into the endogenous $\psi$ loxm 5 locus of the mouse genome (e). PCR analysis of DNAs from mice using L1 and L2 primers from the LacZ gene (f). X-gal staining of $15 \mathrm{dpc}$ fetuses (g). Southern blot analysis of transgene integration using DNA digested with BamHI and XhoI (h). Analysis of sitespecific integration of the transgene by PCR using the indicated primers (G1 and $\mathrm{C} 2$ ) from the endogenous targeted site, and CMV from the transgene (i). Analysis of single-copy and site-specific integration of the transgene by Southern blotting using DNA digested with HaeII (j). DNAs from different tissues of tg1 and 2 mice were extracted and analyzed by PCR for analysis of stable integration of the transgene into various tissues $(\mathbf{k})$. N, non-transgenic; $\mathrm{M}$, size marker; $\mathrm{H}$, heart; K, kidney; L, liver; S, skin; T, testis; 1, lung; m, muscle 
(Fig. 2b). For the $\psi$ loxcorem 5 , a sequence that contains the $13 \mathrm{bp}$ palindromic inverted repeats was derived from loxP, and the $8 \mathrm{bp}$ core was derived from the $\psi$ loxm5. This arrangement was used in order to maximize the binding of Cre recombinase to the palindoromes (Thyagarajan et al. 2000).

Collection of mouse zygotes and DNA microinjection

Seven-week-old B6C3F1 (C57BL/6J X C3H/He) mice purchased from a commercial breeder (Sankyo Laboservice, Tokyo, Japan) were used for the collection of fertilized eggs. Eight-to-ten-week-old ICR female mice were used as foster mothers. Mice were maintained at $22 \pm 2^{\circ} \mathrm{C}$ and $50 \pm 10 \%$ humidity on a 14:10-h light-dark cycle, and provided with food and water ad libitum. $\mathrm{B} 6 \mathrm{C} 3 \mathrm{~F} 1$ females were superovulated by injecting 5 IU each of equine chorionic gonadotropin (eCG) and human chorionic gonadotropin (hCG) at 48-h intervals. Collection of pronuclear stage zygotes and culturing of embryos were carried out according to the standard protocol (Hogan et al. 1994). Pronuclear microinjection of DNA was performed according to the methods used in our previous study (Seo et al. 2000). To test the efficiency of Cre-mediated recombination at an early stage of embryonic development (Fig. 1), $3 \mathrm{ng} / \mu \mathrm{l}$ of pCAG/NCre plasmid and $1 \mathrm{ng} / \mu \mathrm{l}$ of $\mathrm{pCMV} / \mathrm{loxP} / \mathrm{LacZ}$ plasmid were co-injected into the pronucleus of fertilized eggs. To test the endogenous $\psi$ loxm 5 site-specific integration (Fig. 2), $3 \mathrm{ng} / \mu \mathrm{l}$ of pCAG/NCre plasmid and $1 \mathrm{ng} / \mu \mathrm{l}$ of pyloxm5 plasmid were co-injected into the pronucleus of fertilized eggs. The present experiments were conducted according to the guidelines for the use and care of laboratory animals set out by the College of Agriculture, The University of Tokyo.

\section{Analysis of Cre/ $\psi$ loxm 5 recombination}

Genomic DNA was extracted from tissue or embryo. Samples were collected into tubes containing lysis buffer $(10 \mathrm{mM}$ Tris, $50 \mathrm{mM} \mathrm{KCl}, 2.5 \mathrm{mM} \mathrm{MgCl} 2,0.45 \%$ NP-40, $0.45 \%$ Tween $20,60 \mu \mathrm{g} / \mathrm{ml}$ proteinase $\mathrm{K}$ ) and incubated at $55^{\circ} \mathrm{C}$, followed by phenol/chloroform purification. For the analysis of transgenesis, DNAs were analyzed by polymerase chain reaction (PCR) and Southern blotting. For PCR, L1 primer (5'-GCG-TTA-CCC-AAC-TTA-ATC-G-3') and L2 primer (5'-TGT-GAG-CGA-GTA-ACA-ACC-3') were used for amplification of the sequences within the $L a c Z$ gene (Ito et al. 2002; Ito et al. 2005). For Southern blotting, DNA was digested with BamHI and XhoI, electrophoresed, blotted onto membrane, and hybridized with the ${ }^{32} \mathrm{P}$-labeled probe from the lac $Z$ gene. To investigate the site-specific integration of the transgene into the targeted $\psi$ loxm 5 site, DNAs from transgenic mice were analyzed by PCR and
Southern blotting. For PCR, G1 primer (5'-GTT-TAC-TAAATG-CCA-GTG-CTT-TG-3') designed from the endogenous loxm 5 site on the genome (accession No. AF033025) and $\mathrm{C} 1$ primer (5'-CAT-GTA-CTG-GGC-ATA-ATG-CCA-GG-3') from the transgene were used. For Southern blotting, DNA was digested with HaeII located at the 5' and 3' flanking regions of the targeted locus.

\section{Detection of $\beta$ galactosidase ( $\beta$ gal) activity}

X-gal staining was performed according to the methods used in our previous study (Ito et al. 2002; Ito et al. 2005; Ito et al. 2006). The embryos were fixed with $2 \%$ paraformaldehyde in phosphate-buffered saline (PBS)(-) for $15 \mathrm{~min}$. After the embryos had been washed three times with PBS(-) containing $0.05 \%$ bovine serum albumin (BSA), they were transferred to a solution containing $1.2 \mathrm{mM} \mathrm{5-}$ bromo-4-chloro-3-indolyl $\beta$-D-galactopyranoside (Xgal), $2 \mathrm{mM} \mathrm{MgCl}_{2}, 0.1 \%$ Triton X-100, $4 \mathrm{mM} \mathrm{K} \mathrm{K}_{4} \mathrm{Fe}$ $(\mathrm{CN})_{6}$, and $4 \mathrm{mM} \mathrm{K}_{3} \mathrm{Fe}(\mathrm{CN})_{6}$ in PBS(-), then incubated at $30^{\circ} \mathrm{C}$ for $24 \mathrm{~h}$. Beta-gal activity in the embryos was identified by a blue color.

\section{Results}

Cre transient expression and recombination assay in the mouse embryos

Cre has been reported to carry out an integrative recombination in eukaryotic cells (Sauer and Henderson 1990). Cre-mediated integration would be more useful for generating transgenic animals via pronuclear DNA microinjection if recombination occurred at the one-cell stage of embryonic development; recombination at later stages could result in the production of mosaic animals. To monitor the efficiency of Cre-mediated recombination at the one-cell stage of embryonic development, a transient expression assay was conducted. pCMV/LoxP/LacZ (Fig. 1a) and Cre-expressing plasmid pCAG/NCre (Fig. 1b) were microinjected into the pronucleus of fertilized eggs and allowed to develop in M16 medium for $10 \mathrm{~h}$ before X-gal staining. After $10 \mathrm{~h}$ of culturing, 49 embryos were still at the one-cell stage, while 81 embryos were cleaved into the two-cell stage. After X-gal staining (Fig. 1d), 23\% (11/49) and 65\% (53/81) of the embryos were positive at the one-cell and two-cell stages, respectively. The specificity of X-gal staining was confirmed by the fact that embryos injected with $\mathrm{pCMV} / \mathrm{loxP} / \mathrm{LacZ}$ alone were not stained at all. These results suggest that Cre expression and Cre-mediated recombination at loxP sites occurred at the one-cell stage of embryonic development. 
Genomic $\psi$ loxm 5 site-targeting strategy

As depicted in the experimental outline of Fig. 2, the Cremediated integration of the transgene requires two recombination steps. In the first step, the transgene (CMV/LacZ) is integrated into with vector backbone. In the second step, Cre is expected to catalyze excision of the vector backbone and to form hybrid yloxm5. The second recombination should result in a single copy integration of the transgene into the host genome. Out of 16 pups born, four were found to be transgenic by PCR using an L1/L2 primer set (Fig. 2f), a finding that was confirmed by Southern blotting using a probe from the $L a c Z$ gene (Fig. $2 \mathrm{~h}$ ). To assay the expression and the mosaicism of the integrated transgene, other fetuses that had not been allowed to develop to term were stained with X-gal for $\beta$ gal activity at 15 days after the embryo transfer. Out of six fetuses, two exhibited $\beta$ gal activity. One was stained with X-gal over the entire body, and the other showed a mosaic blue deposit in the middle part of the body (Fig. 2g). The transgene integration in Xgal-positive fetuses was confirmed by Southern blotting (Fig. 2h). Next, to investigate whether the transgene was specifically integrated at the endogenous $\psi$ loxm 5 site of the host genome, DNA from the six transgenic mice was analyzed by PCR using specific primer sets, as seen in Fig. 2e. Surprisingly, the 390-bp band was found only in three $(\operatorname{tg} 1, \operatorname{tg} 2, \operatorname{tg} 5)$ of the six transgenic mice (Fig. 2i). Southern blot analysis showed that the correct integration (i.e., site-specific) of the transgene occurred in these three PCR-positive transgenic mice (Fig. 2j). The 4.8-kb bands correspond to a single-copy integrated transgene. These results indicate that the site-specific integration of the transgene occurred in $50 \%$ of the produced transgenic mice. To investigate whether the targeting vector was stably integrated into various tissues of the produced transgenic mice, DNAs were extracted from different tissues and analyzed by PCR. The results showed a mosaic integration of the transgene into different tissues (Fig. 2k).

\section{Discussion}

We have shown that the naturally occurring $\psi$ loxm 5 site of the mouse genome is a functional sequence for the Cremediated DNA recombination event in an embryonic environment. We were able to target the endogenous yloxm5 locus with a high integration frequency for sitespecific integration of the transgene. The transgenesis efficiency in the newborn pups and fetuses that had not been to develop to term was $27 \%$. In the present study, the DNA used for microinjection was in a circular form. The integration efficiency of supercoiled DNA following its injection into the pronucleus has been reported to be very low (5.2\%) (Brinster et al. 1985). Therefore, this result confirmed that Cre recombinase has the potential to promote insertion of foreign DNA into the defined site of the host genome. One of two X-gal-positive fetuses exhibited the blue deposit throughout its whole body; while the other showed mosaic staining with X-gal only in the middle part of its body (Fig. 2g). Mosaic transgenesis was also observed by PCR analysis of different tissues from tg 1 and tg2 (Fig. 2k). This mosaic result would have been caused by the delayed integration of trangene after the onecell stage of embryonic development or as a result of a second-round recombination of $\psi$ loxm 5 -flanked transgene after its integration. Cre protein may still be produced from the Cre-expressing vector, and the newly inserted DNA surrounded by the $\psi$ loxm 5 sequences is subjected again to excision. It has been reported that after transfecting $\mathrm{CHO}$ cells with Cre-expressing plasmid, Cre recombinase is active for 3 days (Koresawa et al. 2000). Fifty percent of our transgenic mice showed the correct integration of the transgene into the targeted $\psi$ loxm 5 locus, which suggests that our trangene was randomly integrated somewhere in the genome of the other three transgenic mice, or that the transgene was specifically integrated into other unknown lox-like sequences in the genome. Loonstra et al. (2001) have reported that chromosomal analysis after Cre expression in mammalian cells revealed numerous chromosomal aberrations and an increased number of sister chromatid exchanges, probably due to the occurrence of other functional lox-like sequences in the genome. The Cre/loxP site-directed recombination system has become an important tool for the genetic manipulation of transgenic animals, and may be important in the future as a tool for human gene therapy. The occurrence of the lox-like sequences in the relatively small yeast genome (Sauer 1996) is strongly indicative of the presence of cryptic lox sites in most eukaryotic genomes. Interestingly, cryptic lox sites were identified by in silico analysis in the mammalian genome. Cre-mediated recombination can occur in $E$. coli cells at the loxP sites of BAC/ PAC (Semprini et al. 2007). The identification of functional lox-like sequences in farm animals would be important for exploiting a simple and efficient method for producing transgenic farm animals. Analysis of the lox structure will certainly be important for this purpose. Characterization of lox sequences has been carried out (Lee and Saito 1998; Missirlis et al. 2006; Sheren et al. 2007).

\section{Conclusions}

Our results showed that the Cre/ $\psi$ lox system is an efficient and simple strategy for targeting an endogenous lox-like site of the genome. This system provides an appealing strategy for circumventing the major problems facing 
transgenic animal production such as the position effect of transgene expression and variations in copy number of integrated transgenes. Moreover, this strategy can enhance the overall efficiency of transgenic animal production.

Acknowledgements This work was supported in part by Grants-inAid for Scientific Research from the Ministry of Education, Science, Sports and Culture of Japan.

\section{References}

Albert H, Dale EC, Lee E, Ow DW (1995) Site-specific integration of DNA into wild-type and mutant lox sites placed in the plant genome. Plant J 7:649-659

Araki K, Araki M, Yamamura K (1997) Targeted integration of DNA using mutant lox sites in embryonic stem cells. Nucleic Acids Res 25:868-872

Brinster RL, Chen HY, Trumbauer ME, Yagle MK, Palmiter RD (1985) Factors affecting the efficiency of introducing foreign DNA into mice by microinjecting eggs. Proc Natl Acad Sci USA 82:4438-4442

Chatterjee PK, Shakes LA, Stennett N, Richardson VL, Malcolm TL, Harewood KR (2010) Replacing the wild type loxP site in BACs from the public domain with lox66 using a lox66 transposon. BMC Res Notes 3:38

Hogan B, Beddington R, Costantini F, Lacy E (1994) Manipulating mouse embryos. A laboratory manual, 2nd edn. Cold Spring Harbor Laboratory Press, New York

Ito M, Yokouchi K, Naito K, Endo H, Hakamata Y, Miyazaki J, Tojo $\mathrm{H}$ (2002) In vitro Cre/loxP system in cells from developing gonads: investigation of the Sry promoter. Dev Growth Differ 44:549-557

Ito M, Yokouchi K, Naito K, Endo H, Hakamata Y, Miyazaki J, Tojo $\mathrm{H}$ (2005) Detection of elements responsible for stage- and tissuespecific expression of mouse Sry using an in vitro Cre/loxP system. Biochem Biophys Res Commun 337:264-270

Ito M, Yokouchi K, Yoshida K, Kano K, Naito K, Miyazaki J, Tojo H (2006) Investigation of the fate of Sry-expressing cells using an in vivo Cre/loxP system. Dev Growth Differ 48:41-47

Kameyama Y, Kawabe Y, Ito Y, Kamihira M (2010) An accumulative site-specific gene integration system using Cre recombinasemediated cassette exchange. Biotechnol Bioeng 105:1106-1114

Koresawa Y, Miyagawa S, Ikawa M, Matsunami K, Yamada M, Shirakura R, Okabe M (2000) Synthesis of a new Cre recombinase gene based on optimal codon usage for mammalian systems. J Biochem 127:367-372

Lee G, Saito I (1998) Role of nucleotide sequences of loxP spacer region in Cre-mediated recombination. Gene 216:55-65

Liu WY, Wang Y, Qin Y, Wang YP, Zhu ZY (2007) Site-directed gene integration in transgenic zebrafish mediated by cre recombinase using a combination of mutant lox sites. Mar Biotechnol (NY) 9:420-428

Loonstra A, Vooijs M, Beverloo HB, Allak BA, van Drunen E, Kanaar R, Berns A, Jonkers J (2001) Growth inhibition and DNA damage induced by Cre recombinase in mammalian cells. Proc Natl Acad Sci USA 98:9209-9214

Niwa H, Yamamura K, Miyazaki J (1991) Efficient selection for highexpression transfectants with a novel eukaryotic vector. Gene 108:193-199

Missirlis PI, Smailus DE, Holt RA (2006) A high-throughput screen identifying sequence and promiscuity characteristics of the loxP spacer region in Cre-mediated recombination. BMC Genomics $7: 73$

Sakai K, Miyazaki J (1997) A transgenic mouse line that retains Cre recombinase activity in mature oocytes irrespective of the Cre transgene transmission. Biochem Biophys Res Commun $237: 318-324$

Sauer B (1987) Functional expression of the Cre-lox site-specific recombination system in the yeast Saccharomyces cerevisiae. Mol Cell Biol 7:2087-2096

Sauer B (1992) Identification of cryptic lox sites in the yeast genome by selection for Cre-mediated chromosome translocations that confer multiple drug resistance. J Mol Biol 223:911-928

Sauer B (1996) Multiplex Cre/lox recombination permits selective site-specific DNA targeting to both a natural and an engineered site in the yeast genome. Nucleic Acids Res 24:4608-4613

Sauer B, Henderson N (1988) Site-specific DNA recombination in mammalian cells by the Cre recombinase of bacteriophage P1. Proc Natl Acad Sci USA 85:5166-5170

Sauer B, Henderson N (1990) Targeted insertion of exogenous DNA into the eukaryotic genome by the Cre recombinase. New Biol 2:441-449

Schmidt EE, Taylor DS, Prigge JR, Barnett S, Capecchi MR (2000) Illegitimate Cre-dependent chromosome rearrangements in transgenic mouse spermatids. Proc Natl Acad Sci USA 97:1370213707

Semprini S, Troup TJ, Kotelevtseva N, King K, Davis JR, Mullins LJ, Chapman KE, Dunbar DR, Mullins JJ (2007) Cryptic loxP sites in mammalian genomes: genome-wide distribution and relevance for the efficiency of BAC/PAC recombineering techniques. Nucleic Acids Res 35:1402-1410

Seo BB, Kim CH, Yamanouchi K, Takahashi M, Sawasaki T, Tachi C, Tojo H (2000) Co-injection of restriction enzyme with foreign DNA into the pronucleus for elevating production efficiencies of transgenic animals. Anim Reprod Sci 63:113-122

Sheren J, Langer SJ, Leinwand LA (2007) A randomized library approach to identifying functional lox site domains for Cre recombinase. Nucleic Acids Res 35:5464-5473

Thyagarajan B, Guimars MJ, Groth AC, Calos MP (2000) Mammalian genomes contain active recombinase recognition sites. Gene 244:47-54

Vega MA (1991) Prospects for homologous recombination in human gene therapy. Hum Genet 87:245-253 